\title{
The Difference Between Secondary Electron Imaging In Variable Pressure SEM And Conventional SEM: Can They Ever Be The "Same"?
}

\author{
B.J. Griffin ${ }^{1,2}$, D.C. Joy ${ }^{3}$ and J.R. Michael $^{4}$ \\ 1 Centre for Forensic Science, The University of Western Australia, Crawley, WA Australia 6009 \\ 2 Centre for Microscopy, Characterization and Analysis, The University of Western Australia, \\ Crawley, WA Australia 6009 \\ 3 Center for NanoPhase Materials Science, Oak Ridge National Laboratory, Oak Ridge, \\ 3N 3nt 3r \\ 4 Sandia National Laboratories, PO Box 5800, Albuquerque, NM 87185-0886
}

Secondary electron images obtained using variable pressure SEM (VPSEM) and the elevated pressure SEM (ESEM) have lower resolution than their conventional high vacuum counterparts, as noted by all manufacturers. Anecdotally the images also lack the typical 3-D character of SEM. Modelling [1] indicates a variable but generally high BSE contribution to the VPSEM images however this is also true of chamber-mounted, Everhardt-Thornley style secondary electron detectors.

Analysis of the SE images, using FFT techniques [2], confirm the lower resolution of the VPSEM and ESEM images relative to those collected using in-column and chamber-mounted SED in high vacuum SEM [3] (fig. 1). The scatter of the primary electron beam by gas in the specimen chamber has been generally accepted as the cause of the lower resolution, although there is no specific data in the literature relating specifically to the image resolution. The VPSEM and ESEM images also show a directional sensitivity and the lack of the typical 'edge-highlighting', as noted earlier (fig. 2).

In review of the recent data in this area, it is also noted that:

(i) the high resolution component in SE images is the SE1 signal, a very low energy signal [4],

(ii) in VPSEM conditions, x-ray data show that electron landing energies are increased by up to and $100 \mathrm{v}$ by the presence of a positive ion cloud above the sample surface [5],

(iii) a positive ion sink (widget) above the sample improves imaging quality [6].

A hypothesis is that the positive ion cloud, formed by the electron gas cascade to the positively biased GSE detector, acts as a dynamic energy filter on the emitted SE signal. The degree of loss of the low energy SE will be affected by most operating conditions, as is well described for the gas amplification, because the ion cloud is effectively the reciprocal product of the electron-gas interactions. The higher energy SE and BSE will be unaffected by the ion field strengths.

The hypothesis then suggests that SE images under VPSEM and/or ESEM conditions must be lower resolution, unless the ion cloud effects can be avoided. This effect is relatively independent of sample conductivity as the energy filtering occurs above the surface. One experiment in support of this has been to image an insulator surface at high water pressure and progressively reducing the sample temperature. At the dew point the image brightness increases significantly as a condensed water layer devlops as a conductive layer. This layer then eliminates the suppression of SE emission by (negative) surface charging. However the energy filtering remains as the ion cloud is av=bove the surface and the image detail still does not match conventional SEM.

The model encourages design of new integrated GSE detector - ion sink configurations, to improve the SE performance of VPSEM and ESEM. Current designs will be presented. 
References

(1) A.L. Fletcher et al. (1997) J. Phys. D: Appl. Phys. 30, 2249.

(2) D. C. Joy et al. (2007), Microsc. Microanal., 13,1682

(3) B.J. Griffin et al. (2010), Microsc. Microanal., 16, 624

(4) B.J. Griffin et al. (2009), Microsc. Microanal., 15, 46

(5) M. Toth et al. (2002) J. Appl. Phys. 91, 4479

(6) J.P. Craven et al. (2002) J. Microsc. 205, 96-105.

(7) Sandia is a multiprogram laboratory operated by Sandia Corporation, a Lockheed Martin Company, for the United States Department of Energy (DOE) under contract DEAC0494AL85000.

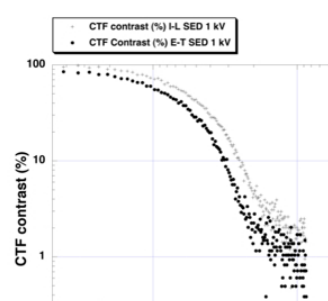

$1 \mathrm{kV}$
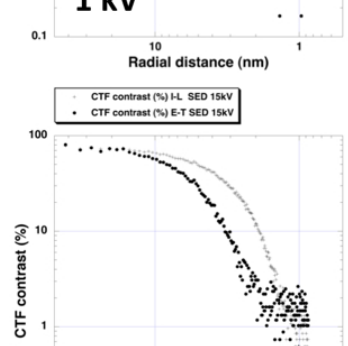

$15 \mathrm{kV}$

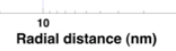

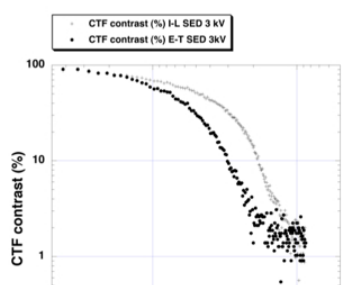

$3 \mathrm{kV}$

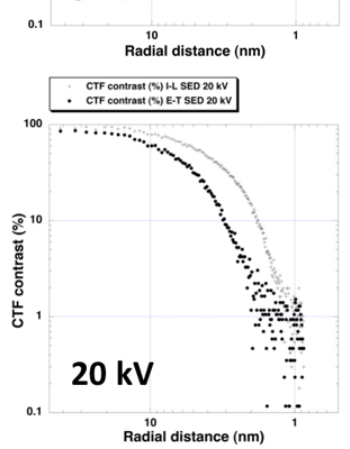

Figure 1(a): CTF comparison of the variations between incolumn and below-column SED image resolution in conventional SEM over a range of accelerating voltage.

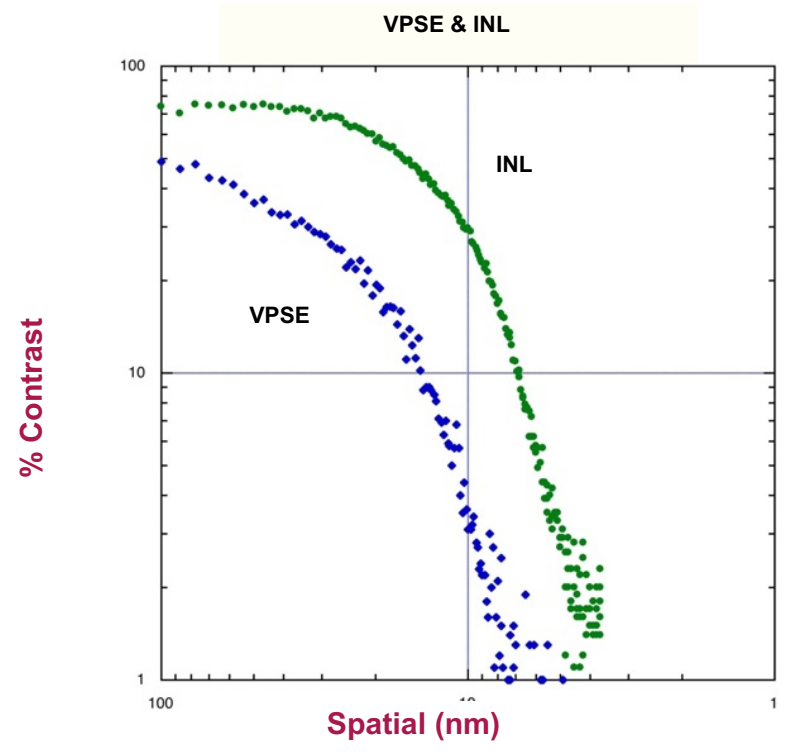

Figure 1(b) CTF comparison between in-column and below-column VPSEM image resolution at $20 \mathrm{kV}$. E-T SED has intermediate performance as per fig. 1(a).

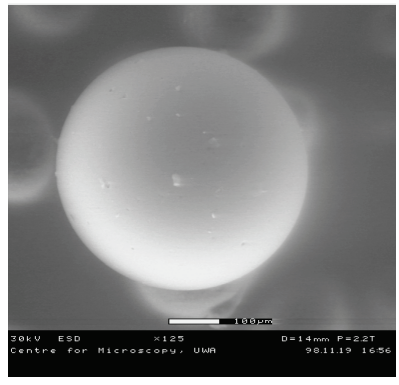

(a) $30 \mathrm{kV}, 2.2$ torr, $\mathrm{H}_{2} \mathrm{O}$ -wire detector@SW

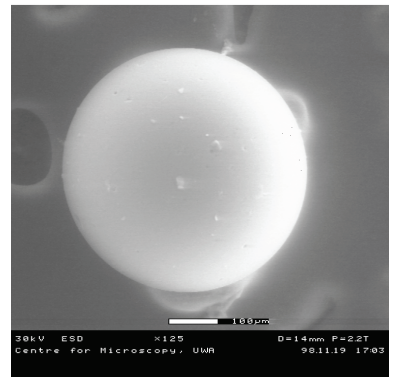

(b) $30 \mathrm{kV}, 2.2$ torr , $\mathrm{H}_{2} \mathrm{O}$ -wire detector@NE

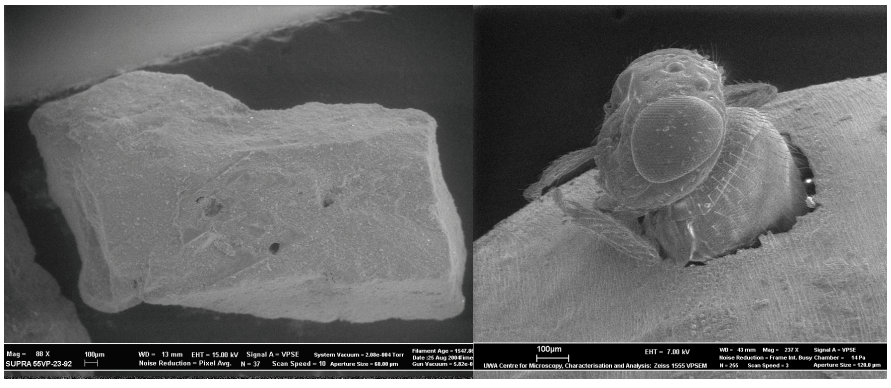

(c) $15 \mathrm{kV}, 0.2$ torr, air, (d) $7 \mathrm{kV}, 0.1$ torr, air - 'kitty litter' grain
- emerging parasitic wasp

Figure 2: SE images of various samples under VPSEM and ESEM conditions illustrating the low '3-D’ nature. 